\title{
Assessment of prescribed medications and pattern of distribution for potential drug-drug interactions among chronic kidney disease patients attending the Nephrology Clinic of Lagos University Teaching Hospital in Sub-Saharan West Africa
}

This article was published in the following Dove Press journal:

Clinical Pharmacology:Advances and Applications

26 October 2017

Number of times this article has been viewed

\author{
Olumuyiwa John Fasipe' \\ Peter Ehizokhale Akhideno ${ }^{2}$ \\ Obiyo Nwaiwu ${ }^{3}$ \\ Alex Adedotun Adelosoye ${ }^{4}$ \\ 'Department of Pharmacology \& \\ Therapeutics, University of Medical \\ Sciences, Ondo City, Ondo State, \\ ${ }^{2}$ Department of Internal Medicine, \\ Irrua Specialist Teaching Hospital, \\ Irruar, Edo State, ${ }^{3}$ Department \\ of Pharmacology \& Therapeutics, \\ University of Lagos, Yaba, Lagos State, \\ ${ }^{4}$ Department of Family Medicine, \\ University of Medical Sciences, Ondo \\ City, Ondo State, Nigeria
}

Correspondence: Olumuyiwa John Fasipe Department of Pharmacology \& Therapeutics, University of Medical Sciences, Laje Road, Ondo City, Ondo State, Nigeria

Tel +2348035098261

Email fasipe.olumuyiwa@yahoo.com
Introduction: Life expectancy has increased significantly among chronic kidney disease (CKD) patients due to the extensive use of polypharmacy practice for medication prescriptions. This predisposes them to potential drug-drug interactions (DDIs), which can lead to an increase in morbidity, mortality, length of hospital stay, and health care cost.

Methods: This was a 30-month retrospective study that reviewed the medical case records of consenting adult CKD patients from January 2014 to June 2016. The Medscape drug reference database was used to evaluate patients' medications for potential DDIs.

Results: This study involved 123 adult CKD patients (63 [51.22\%] males and 60 [48.78\%] females) with a mean age of $53.81 \pm 16.03$ years. The most common comorbid conditions were hypertension (112 [91.10\%]) and diabetes mellitus (45 [36.60\%]). Regarding the form of nephrological interventions being offered, the majority of the respondents - 66 (53.66\%) were on maintenance dialysis, followed by $53(43.09 \%)$ respondents on conservative care, while 4 (3.25\%) respondents were on renal transplantation. A total of 1264 prescriptions were made, and the mean number of prescribed medications per patient was $10.28 \pm 3.85$. The most frequently prescribed medications were furosemide (88 [71.6\%]), heparin (67 [54.47\%]), lisinopril (65 [52.9\%]), oral calcium carbonate $\left(\mathrm{CaCO}_{3}\right)(63$ [51.2\%]), $\alpha$-calcidol (62 [50.4\%]), and erythropoietin (61 [49.6\%]). A total number of 1851 potential DDIs were observed among 118 patients. The prevalence of potential DDIs in this study was $78.0 \%$, while the mean DDI per prescription was 1.50. Among the potential DDIs observed, the severity was mild in 639 (34.5\%) patients, moderate in $1160(62.7 \%)$ patients, and major in $51(2.8 \%)$ patients and only $1(0.1 \%)$ patient was of contraindicated drug combination. The most frequent DDIs' pattern observed was between oral $\mathrm{CaCO}_{3}$ and oral ferrous sulfate. There was a statistically significant association between the number of prescribed medications and the estimated glomerular filtration rate (eGFR; pre-ESRD and ESRD staging) with a $P$-value of 0.00000119 . This implies that the number of prescribed medications increases as the eGFR declines in advance CKD stage patients. Conclusion: Most of these interactions have moderate severity and delayed onset, hence the need to follow-up these patients after prescription in order to reduce associated morbidity, mortality, length of hospital stay, and health care cost. Physicians and clinical pharmacists should utilise available interaction software to avoid harmful DDIs in these patients.

Keywords: potential, drug-drug interactions, chronic kidney disease, polypharmacy, prescribed medications, pattern of distribution, Medscape interaction checker 


\section{Introduction}

Chronic kidney disease (CKD) can be defined as a progressive and irreversible deterioration in the renal function of an individual over a period of at least 3 months regardless of the underlying etiology. ${ }^{1}$ In Nigeria and worldwide, poorly controlled chronic hypertension, poorly controlled diabetes mellitus, and HIV-associated nephropathy among others are the common causes of $\mathrm{CKD} .{ }^{2,3}$ Although efficacy, compliance, and economic factors are considered in selecting drugs for treating patients with CKD, these patients are usually on polypharmacy (ie, taking more than five different drugs for the treatment of a particular disease or group of diseases) with attendant risk for drug-drug interactions (DDIs) and adverse drug reactions (ADRs) ${ }^{3,4}$ The influences of the disease on drugs' pharmacokinetic and pharmacodynamics parameters increase the risk for DDIs and ADRs; these further compound the problems of patients with CKD and may further increase morbidity, mortality, length of hospital stay, and health care cost among them. ${ }^{5,6}$

CKD is a major public health problem due to its increasing incidence, prevalence, and associated high burden. The global prevalence of CKD is estimated to be $11-13 \% .{ }^{7,8}$ The prevalence of CKD in Nigeria varied between $11.4 \%$ and $18.8 \%$ from both community- and hospital-based studies. ${ }^{2-5}$ Cardiovascular disease burden in CKD patients is high and associated with increased hospitalization, morbidity, and mortality. ${ }^{6-8}$

Cardiovascular risk factors such as hypertension, diabetes mellitus, anemia, calcium-phosphate abnormalities, hyperuricemia, and left ventricular hypertrophy are highly prevalent in $\mathrm{CKD} .{ }^{9,10}$ These are largely responsible for cardiovascular disease burden and some complications in CKD patients. Management of these cardiovascular diseases and risk factors is important in retarding progression of CKD and reducing mortality. ${ }^{79}$ This involves the use of multiple drug combination therapy in the management of CKD and its attendant complication, and hence polypharmacy is often practiced. The consequences of polypharmacy include poor patients' medication compliance due to high pill burden, increased cost of care, and most importantly DDI, which may have deleterious effects on patients.

DDI can be defined as an appreciably harmful or beneficial process whereby the pharmacological effect of a drug is directly or indirectly influenced and modified by the presence of another drug, which can result in either treatment failure (antagonistic interaction) or drug-induced toxicity (synergistic/additive interaction). ${ }^{11}$ It may result from a number of processes, which is related to either pharmacokinetic or pharmacodynamic properties of the drugs. Consequences of
DDIs include increase or decrease in the efficacy of treatment and ADRs.

DDIs are major clinical problem, accounting for $2-6 \%$ of all hospital admissions with estimated annual cost to the National Health Service of $\sim £ 500$ million in the UK. ${ }^{12}$ In a meta-analysis of 39 prospective studies from hospital in the USA, it was shown that DDIs ranked fourth to sixth as a leading cause of death. ${ }^{13} \mathrm{Al}-\mathrm{Hajje}$ et a ${ }^{14}$ also reported that drug interactions were the most common drug-related problems in hospitalized medical patients in the University Hospital of Beirut.

The prevalence of potential DDIs in CKD patients from previous reports ranged between $76.1 \%$ and $89.1 \% .{ }^{15-20}$ This relatively high prevalence is related to the polypharmacy that is involved in the management of this condition. It is associated with increased morbidity, mortality, length of hospital stay, and health care cost. ${ }^{21-25}$ These added burdens of harmful DDIs and its consequences in CKD patients are preventable because of their predictable nature. Various software are available that can detect potential DDIs such as British National Formulary, Drug Interaction Facts, Medscape, Epocrates, Lexi-Interact, Harmavista, and Stockley's Drug Interactions.

The clinical pharmacists also have major role in preventing DDI by evaluating physicians' prescriptions for possible DDI. ${ }^{14,26}$ Therefore, integrated professional interaction should be encouraged between nephrologist/physicians and clinical pharmacists in order to optimize CKD patients' care. Vigilance by health care workers such as clinicians, pharmacists, and nurses in detecting, diagnosing, and reporting drug interactions, particularly in at risk individuals such as CKD patients, is also vital for continued drug safety monitoring.

This study was designed to assess the prescribed medications and pattern of distribution for potential DDIs among CKD patients attending the Nephrology Clinic of Lagos University Teaching Hospital (LUTH) in Sub-Saharan West Africa. This will create awareness on the burden of potential DDIs in renal practice and will bring to limelight the need to regularly evaluate prescriptions of CKD patients for any medication-related problems.

\section{Methods}

This study was a retrospective study carried out at the Nephrology Clinic of LUTH in Sub-Saharan West Africa. It receives referral from within and outside the state.

A total of 123 consented adult CKD patients who were being managed at the center over a 30-month period between January 2014 and June 2016 were recruited for the study. Patients below the age of 18 years, those being managed 
for acute kidney injury, and adult CKD patients who did not grant their informed consent were excluded from the study. The medical case records of all the adult CKD patients were retrieved after a verbal informed consent was obtained from each of them, and the following information was extracted using a pro forma: sociodemographic data, stage of CKD, number and list of medications at the time of last clinic visit for outpatients and at the time of discharge for those who received in-patient care, number and list of comorbidities such as hypertension, diabetes mellitus, HIV infection, stroke, and heart failure. The serum creatinine was used to calculate the estimated glomerular filtration rate (eGFR) using Chronic Kidney Disease Epidemiology Collaboration (CKD-EPI) formula, and CKD staging was done using eGFR as follows: stage 1 (eGFR of $\geq 90 \mathrm{~mL} / \mathrm{min}$ with evidence of kidney damage), stage 2 (eGFR of $60-89 \mathrm{~mL} / \mathrm{min}$ with or without evidence of kidney damage), stage 3 (eGFR of $30-59 \mathrm{~mL} / \mathrm{min}$ with or without evidence of kidney damage), stage 4 (eGFR of 15-29 mL/min with or without evidence of kidney damage), and stage 5 (eGFR $<15 \mathrm{~mL} / \mathrm{min}$ with or without evidence of kidney damage). ${ }^{27}$

The Medscape drug reference database was used to evaluate patients' medication regimen for potential DDIs. Medscape drug reference database has been reported to show the best results in precision analysis. ${ }^{28}$ The Medscape drug reference database system provides accurate information about the risk, type, mechanism, and pattern of distribution of potential DDIs. It also gives recommendation(s) on how to prevent and manage DDIs if they occur. The software identifies and classifies DDIs according to their level of clinical significance in categories. These categories are types A, B, C, D, and X:

1. Type A: no known interaction

2. Type B: minor or mild interaction

3. Type $\mathrm{C}$ : moderate or significant interaction

4. Type D: major or serious interaction

5. Type $\mathrm{X}$ : contraindication or avoid combination

The mean potential DDI per prescription was obtained by dividing the total number of DDIs by the total number of prescriptions.

\section{Ethical consideration and maintenance of participants' confidentiality}

Ethical clearance for the study was obtained from the Health Research Ethical Committee of LUTH.

In addition, a verbal informed consent was obtained from each of the adult CKD patients whose medical case records were used, while the medical case records of those who did not grant their informed consent were excluded from the study. Participants' confidentiality were respected and maintained by ensuring that no unauthorized person has access to the information on the questionnaires, no information can be traced to the respondents (as numbering system was used for the questionnaires instead of writing the patients' names on them), and no unauthorized use of information was made. Effort was made to ensure that no harm was done to the respondents. Those who were at risk of harmful potential DDIs were monitored closely, and feasible alternative prescriptions were made where the concomitant risk and adverse effects outweigh the benefits.

\section{Data analysis}

Data generated were analyzed using the statistical package for social sciences version 17 (released 2008; SPSS Inc., Chicago, IL, USA). Results were presented in tabular form. Discrete variables were presented as frequency and percentages. Continuous variables were presented as mean \pm standard deviation.

\section{Results}

This study involved 123 CKD patients (63 [51.22\%] males and $60[48.78 \%]$ females). The mean age of the study subjects was $53.81 \pm 16.03$ years. A total of $48(39.0 \%)$ patients were between 18 and 49 years, $52(42.3 \%)$ patients were between 50 and 69 years, and the remaining $23(18.7 \%)$ patients were 70 years and older (Table 1 ).

A total of $53(43.09 \%)$ study subjects had tertiary education, 36 (29.3\%) subjects had secondary education, 18 $(14.6 \%)$ subjects had primary education, and $16(13.0 \%)$ subjects had no formal education. A total of $86(69.9 \%)$ study subjects were in CKD stage 5, 15 (12.2\%) subjects were in CKD stage 4, 19 (15.5\%) subjects were in CKD stage 3, 2 (1.6\%) subjects were in CKD stage 2 , and the remaining 1 subject was in CKD stage 1 (Table 1).

Regarding the form of nephrological interventions being offered, majority of the respondents - $66(53.66 \%)$ were on maintenance dialysis, followed by 53 (43.09\%) subjects on conservative care, while $4(3.25 \%)$ subjects were on renal transplantation (Table 1).

The most common comorbid conditions were hypertension in $112(91.10 \%)$ patients and diabetes mellitus in 45 $(36.60 \%)$ patients. Other conditions were heart failure in 11 $(8.9 \%)$ patients, HIV in $7(5.7 \%)$ patients, and stroke in 5 (4.1\%) patients (Table 1 ).

A total number of 1264 medications were prescribed for the study subjects, and the mean number of prescribed medi- 
Table I Characteristics of study population

\begin{tabular}{ll}
\hline Characteristics & $\mathbf{N}(\%) /$ mean \pm SD \\
\hline Gender & \\
Male & $63(51.22)$ \\
Female & $60(48.78)$ \\
Mean age (years) & $53.81 \pm 16.03$ \\
Age group & \\
$20-49$ years & $48(39.0)$ \\
$50-69$ years & $52(42.3)$ \\
$\geq 70$ years & $23(18.7)$ \\
Level of education & \\
No formal education & $16(13.0)$ \\
Primary & $18(14.6)$ \\
Secondary & $36(29.3)$ \\
Tertiary & $53(43.1)$ \\
CKD stage & \\
I & $1(0.8)$ \\
2 & $2(1.6)$ \\
3 & $19(15.5)$ \\
4 & $15(12.2)$ \\
5 & $86(69.9)$ \\
Comorbidities & \\
Hypertension & $112(91.10)$ \\
Diabetes mellitus & $45(36.60)$ \\
Heart failure & $11(8.9)$ \\
HIV & $7(5.7)$ \\
Stroke & $5(4.1)$ \\
Mean prescribed medication per patient & $10.28 \pm 3.85$ \\
Number of prescribed medications & \\
$\leq 5$ & $18(14.6)$ \\
$6-10$ & $43(35.0)$ \\
II-I5 & $51(41.5)$ \\
Morm of nephrological interventions & $11(8.9)$ \\
Conservative care & $66(53.66)$ \\
Renal transplantation & $53(43.09)$ \\
\hline Ab & $4(3.25)$ \\
\hline
\end{tabular}

Abbreviations: CKD, chronic kidney disease; SD, standard deviation.

cations per patient was $10.28 \pm 3.85$. A total of $18(14.6 \%)$ CKD subjects were on $\leq 5$ medications, $43(35.0 \%)$ subjects were on 6-10 medications, 51 (41.5\%) subjects were on $11-15$ medications, and 11 (8.9\%) subjects were on $\geq 16$ medications (Table 1).

The most frequently prescribed medications were furosemide (88 [71.6\%]), heparin (67 [54.47\%]), lisinopril (65 [52.9\%]), oral calcium carbonate $\left(\mathrm{CaCO}_{3}\right)(63$ [51.2\%]), $\alpha$-calcidol (62 [50.4\%]), erythropoietin (61 [49.6\%]), intravenous iron sucrose (60 [48.78\%]), amlodipine (56 [45.5\%]), hydrochlorthiazide (53 [43.1\%]), folic acid (53 [43.1\%]), and oral ferrous sulfate (OFS) (50 [40.7\%]) (Table 2).

A total number of 1851 potential interactions were identified among 118 (95.9\%) subjects while 5 (4.1\%) subjects had no known interaction (type A). The mean DDI
Table 2 List of most frequently prescribed medications

\begin{tabular}{ll}
\hline Medications & N (\%) \\
\hline Furosemide & $88(71.6)$ \\
Heparin & $67(54.47)$ \\
Lisinopril & $65(52.9)$ \\
Oral calcium carbonate & $63(51.22)$ \\
Alfacalcidol & $62(50.4)$ \\
Erythropoeitin & $61(49.6)$ \\
Intravenous iron sucrose & $60(48.8)$ \\
Amlodipine & $56(45.5)$ \\
Hydrochlorthiazide & $53(43.1)$ \\
Folic acid & $53(43.1)$ \\
Oral ferrous sulfate & $50(40.7)$ \\
Astyfer (multivitamins) & $47(38.2)$ \\
Vitamin C & $40(32.5)$ \\
Alpha-methyldopa & $32(26.0)$ \\
Atorvastatin & $30(24.4)$ \\
Insulin & $25(20.3)$ \\
Valsartan & $24(19.5)$ \\
\hline
\end{tabular}

per prescription was 1.5 , while the prevalence of potential DDIs was $78.0 \%$. Twenty-nine (23.6\%) subjects had 6-10 interactions, $26(21.1 \%)$ subjects had 11-15 interactions, while 16 (13.0\%) subjects had 21-25 interactions (Table 3). The range for the number of potential interactions among the 118 respondents was $1-85$ interactions with a mean value of $15.69 \pm 11.00$ interactions per patient.

A total of 639 (34.5\%) subjects were of mild severity (type B), 1160 (62.7\%) subjects were of moderate severity (type C), 51 (2.8\%) subjects were of major severity (type D), and only $1(0.1 \%)$ subject was of contraindicated/avoid drug combination (type X) (Table 4). The contraindicated/avoid drug combination occurred between intravenous calcium gluconate and intravenous ceftriaxone.

A total of 960 (52\%) DDIs were pharmacokinetic interactions, while the remaining 891 (48\%) DDIs were pharmacodynamic interactions (Figure 1).

Regarding the pattern of distribution for DDIs among the respondents, a total number of 405 different patterns of interactions were observed with the most frequent DDIs' pattern occurring between oral $\mathrm{CaCO}_{3}$ and OFS (184 [9.94\%]), which belonged to pharmacokinetic type $\mathrm{B}$ and type $\mathrm{C}$ interaction categories. Other frequently occurring patterns of interactions were between folic acid and furosemide (63 [3.40\%]), $\alpha$-calcidol and $\mathrm{CaCO}_{3}(60$ [3.24\%]), OFS and vitamin $\mathrm{E}$ (56 [3.03\%]), furosemide and lisinopril (49 [2.65\%]), $\mathrm{CaCO}_{3}$ and furosemide (49 [2.65\%]), furosemide and hydrochlorthiazide (HCTZ) (42 [2.27\%]), heparin and vitamin E (35 [1.89\%]), folic acid and HCTZ (35 [1.89\%]), heparin and lisinopril (34 [1.84\%]), amlodipine and $\mathrm{CaCO}_{3}(32[1.73 \%])$, and $\mathrm{CaCO}_{3}$ and lisinopril (31 [1.67\%]) (Table 5). 
Table 3 Number of drug-drug interactions (DDIs), and test for associations between parameters for the study population

(A) Number of drug-drug interactions (DDIs) for the study population

\begin{tabular}{ll}
\hline Number of DDIs & $\mathbf{N}(\%)$ \\
\hline None & $\mathbf{5}(\mathbf{4 . 1 \% )}$ \\
\hline $1-5$ & $15(12.2 \%)$ \\
$6-10$ & $29(23.6 \%)$ \\
$11-15$ & $26(21.1 \%)$ \\
$16-20$ & $15(12.2 \%)$ \\
$21-25$ & $16(13.0 \%)$ \\
$\geq 26$ & $17(13.8 \%)$ \\
\hline
\end{tabular}

(B) Test for association between the number of prescribed medications and the estimated glomerular filtration rate (eGFR) for the study population

\begin{tabular}{lll}
\hline & $\begin{array}{l}\text { Pre-ESRD Stage } \\
\text { (eGFR } \geq 15 \mathrm{ml} / \mathbf{m i n})\end{array}$ & $\begin{array}{l}\text { ESRD Stage } \\
\text { (eGFR 0-14ml/min) }\end{array}$ \\
\hline I -5 Medications & 13 & 5 \\
$6-10$ Medications & 18 & 25 \\
II -15 Medications & 5 & 46 \\
$\geq 16$ Medications & 1 & 10 \\
\hline
\end{tabular}

(C) Test for association between the number of prescribed medications and the occurrence of DDls among the study population

\begin{tabular}{lll}
\hline & DDls present & DDIs absent \\
\hline I-5 medications & $\mathrm{I} 4$ & 4 \\
$\geq 6$ medications & 104 & $\mathrm{I}$ \\
\hline
\end{tabular}

Notes: (A) Mean DDIs $=15.69 \pm 1 \mathrm{I} .00$ interactions per patient. (B) Chi- $X^{2}=30.3 \mathrm{I}$, df $=3, P$-value $=0.00000119$ (significant), critical value $=7.815, \alpha=0.05$. (C)Chi- $X^{2}$ $=17.83, \mathrm{~d} f=\mathrm{I}, P$-value $=0.0000242 \mathrm{I}$ (significant), critical value $=3.84 \mathrm{I}, \alpha=0.05$.

Abbreviation: ESRD, end stage renal disease.

Table 4 Severity of drug-drug interactions

\begin{tabular}{ll}
\hline Severity of DDIs & Frequency (\%) \\
\hline Type B (mild severity) & $639(34.5)$ \\
Type C (moderate severity) & $1160(62.7)$ \\
Type D (major severity) & $5 \mathrm{I}(2.7)$ \\
Type X (contraindicated) & $\mathrm{I}(0.1)$ \\
\hline
\end{tabular}

Abbreviation: DDIs, drug-drug interactions.

In this study, there was a statistically significant association between the number of prescribed medications and the eGFR (pre-end stage renal disease [pre-ESRD] and ESRD staging) for the respondents with a $P$-value of 0.00000119 . This implies that the number of prescribe medications increases as the eGFR declines in advance CKD stage patients (Table 3 ). In addition, there was also a statistically significant association between the number of prescribed medications and the occurrence of DDIs among the respondents with a P-value of 0.00002421 . This implies that as the number of prescribe medications increases the chances for occurrence of DDIs increases among these CKD patients. About $77.8 \%$ of those CKD patients taking around 1-5 medications are at risk of developing drug-drug interactions while $99.05 \%$ of those taking around 6 or more ( $\geq 6$ ) medications are at risk of developing DDIs (Table 3).

\section{Discussion}

In this study, the mean prescribed medications per patient was $10.28 \pm 3.85$. This is slightly higher than $7.87 \pm 2.44$ reported by Al-Ramahi et al. ${ }^{17}$ However, Rama et $a{ }^{16}$ reported a higher mean prescribed drugs per patient of $12.08 \pm 6.30$ compared to the finding in this present study. Our study population involved both nondialyzed and dialyzed patients unlike the study by Al-Ramahi that involved only CKD patients on maintenance hemodialysis (MHD). Patients who are on regular MHD may require less number of drugs for blood pressure control; hence, this may possibly explain the lower mean number of prescribed medications. The practice of polypharmacy in the management of CKD is however not surprising because they have high number of cardiovascular risk factors, comorbidities, and complications such as hypertension, diabetes mellitus, heart failure, arrhythmias, anemia, hyperuricemia, and calcium-phosphate abnormalities, which are managed by a combination of drugs.

The most common comorbidities in this study were hypertension and diabetes, which agreed with previous studies. ${ }^{19,20,29}$ This can be attributed to the fact that both conditions are the leading etiologies of CKD in Nigeria, Sub-Saharan West Africa region, and worldwide.

This study showed that the prevalence of potential DDIs among the studied CKD patients is $78.0 \%$ with $\sim 62.7 \%$ showing moderate clinical significance. Most of the DDIs had delayed onset and would require long-time follow-up in order to actually determine the clinically significant outcome of these interactions. The average number of DDIs per prescription in this study was 1.5, which is similar to the report by Sgnaolin et $a \mathrm{l}^{15}$ (1.0) but lower than the number reported by Rama et $\mathrm{al}^{16}$ (2.7) and Marquito et $\mathrm{al}^{19}$ (2.5). This showed that there was likelihood of at least a potential DDI for each prescription issued out to a CKD patient in this study. This therefore emphasized the need for physicians and clinical pharmacists to regularly evaluate prescriptions of CKD patients for DDI.

The prevalence of potential DDIs in this study was $78.0 \%$, which fell in between the range of $76.1-89.1 \%$ reported in some previous studies. ${ }^{16-20}$ However, the reported prevalence in this study was higher than $56.9 \%$, which was reported by Sgnaolin et al. ${ }^{15}$

Differences in methodology, average number of medications per prescription, and CKD stage of the studied population could account for variation in the prevalence rates of potential DDIs in the different studies. For example, in the study by Sgnaolin et $\mathrm{al}^{15}$ where a lower prevalence of potential DDIs was reported compared to this study, the average number of medications per prescription was $6.3 \pm 3.1$ unlike 


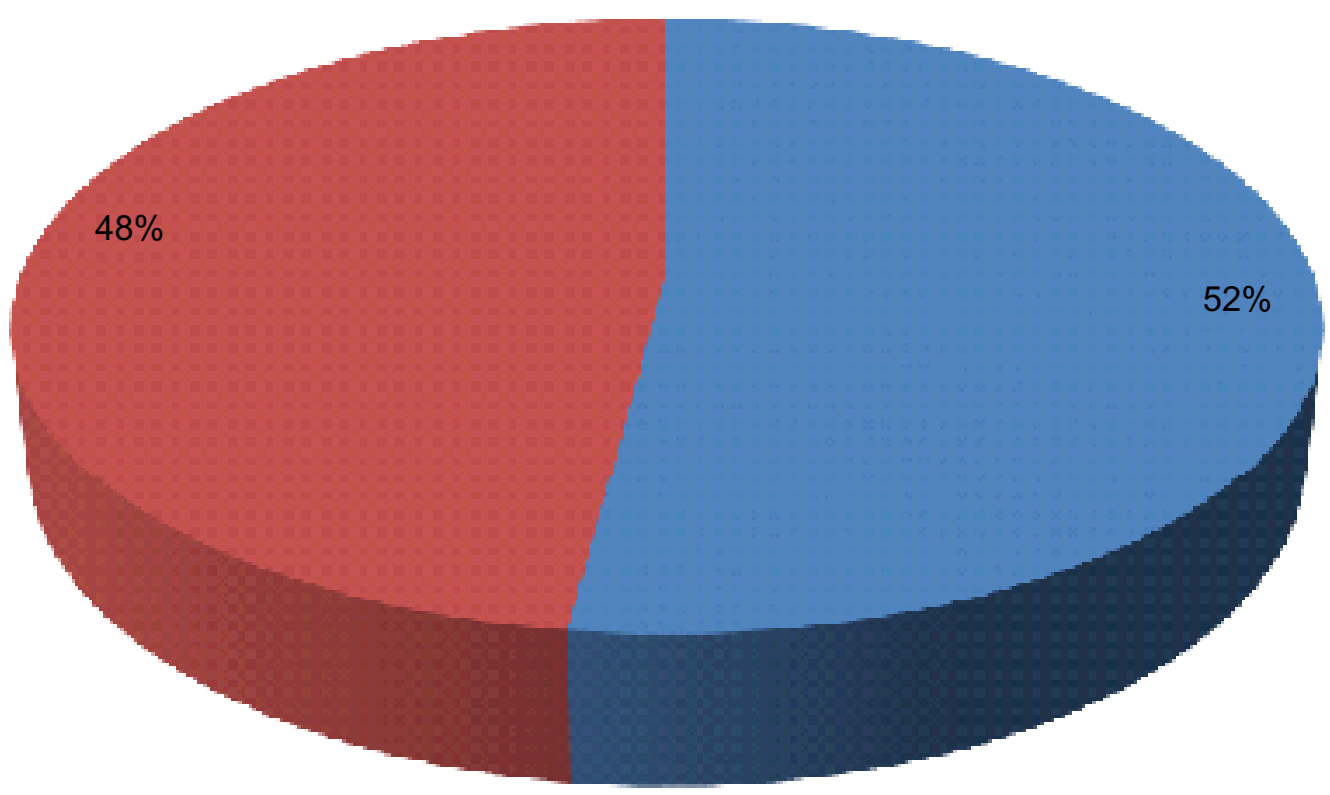

Pharmacokinetic interaction

- Pharmacodynamic interaction

Figure I Mechanism of drug-drug interactions in the study population.

Table 5 Pattern of distribution for common specific drug-drug interactions and their potential adverse effects

\begin{tabular}{|c|c|c|c|c|}
\hline $\begin{array}{l}\text { Pattern of specific drug } \\
\text { interactions }\end{array}$ & $\mathbf{N}$ & $\%$ & Mechanism of interaction & Potential adverse effects \\
\hline $\mathrm{CaCO}_{3}+\mathrm{OFS}$ & 184 & 9.94 & Pharmacokinetic, types B and C & $\begin{array}{l}\mathrm{CaCO}_{3} \text { will decrease intestinal absorption of OFS by increasing } \\
\mathrm{GIT} \mathrm{pH} \text { and vice versa }\end{array}$ \\
\hline Folic acid + furosemide & 63 & 3.40 & Pharmacokinetic, type B & Furosemide increases renal clearance of folic acid \\
\hline$\alpha$-Calcidol $+\mathrm{CaCO}_{3}$ & 60 & 3.24 & Pharmacokinetic, type C & Hypercalcemia \\
\hline OFS + vitamin E & 56 & 3.03 & Pharmacokinetic, type C & Vitamin E decreases GIT absorption of OFS \\
\hline $\mathrm{CaCO}_{3}+$ furosemide & 49 & 2.65 & Pharmacokinetic, type B & Furosemide increases renal clearance of calcium \\
\hline Furosemide + lisinopril & 49 & 2.65 & Pharmacodynamics, type C & Acute hypotension and renal insufficiency \\
\hline Furosemide $+\mathrm{HCTZ}$ & 42 & 2.27 & Pharmacodynamics, type C & Hypokalemia, acute hypotension, and renal insufficiency \\
\hline Folic acid + HCTZ & 35 & 1.89 & Pharmacokinetic, type B & HCTZ increases renal clearance of folic acid \\
\hline Heparin + Vitamin E & 35 & 1.89 & Pharmacodynamics, Type B & $\begin{array}{l}\text { Increase risk of bleeding/hemorrhage due to their anticoagulant } \\
\text { effect }\end{array}$ \\
\hline Heparin + lisinopril & 34 & 1.84 & Pharmacodynamics, type C & $\begin{array}{l}\text { Low molecular weight heparin may suppress adrenocortical } \\
\text { aldosterone secretion, thereby leading to hyperkalemia }\end{array}$ \\
\hline Amlodipine $+\mathrm{CaCO}_{3}$ & 32 & 1.73 & Pharmacodynamics, type C & $\begin{array}{l}\mathrm{CaCaO}_{3} \text { antagonizes and decreases the vasodilatory effect } \\
\text { of amlodipine on the small arteries, thereby reducing the } \\
\text { antihypertensive effect }\end{array}$ \\
\hline $\mathrm{CaCO}_{3}+$ lisinopril & 31 & 1.67 & Pharmacodynamics, type C & $\begin{array}{l}\mathrm{CaCaO}_{3} \text { antagonizes and decreases the vasodilatory effect } \\
\text { of lisinopril on the small arteries, thereby reducing the } \\
\text { antihypertensive effect }\end{array}$ \\
\hline
\end{tabular}

Abbreviations: GIT, gastrointestinal tract; HCTZ, hydrochlorthiazide; OFS, oral ferrous sulfate.

this present study where the average number of medications per prescription was $10.28 \pm 3.85$. The number of medications per prescription has been reported as one of the major determinants of potential DDIs. ${ }^{15,18,20}$

The most frequently prescribed medications in this study were furosemide, heparin, lisinopril, and oral $\mathrm{CaCO}_{3}$. This agreed with previous studies where furosemide and oral
$\mathrm{CaCO}_{3}$ were also reported as among the most frequently prescribed medications for CKD patients. ${ }^{17,19}$

The most frequent DDI in this study was between oral $\mathrm{CaCO}_{3}$ and OFS. The intestinal absorption of oral iron may be reduced when coadministered with oral $\mathrm{CaCO}_{3}$ because of the effect of the latter in increasing the gastrointestinal $\mathrm{pH}$. This is similar to findings from studies by Hedge et al and 
Sgnaolin et al. ${ }^{15,20}$ They reported interactions between oral $\mathrm{CaCO}_{3}$ and OFS as among the most frequently occurring DDIs in their studies. However, report from this present study differs from that of Rama et al ${ }^{16}$ and Al-Ramahi et al. ${ }^{17}$ Rama et al ${ }^{16}$ reported ascorbic acid and cyanocobalomin, while Al-Ramahi et $\mathrm{al}^{17}$ reported $\mathrm{CaCO}_{3}$ and amlodipine as the most frequent interaction in their respective studies. Furthermore, interactions between $\mathrm{CaCO}_{3}$ and some antihypertensives such as lisinopril and amlodipine observed in this study may also lead to a reduction in their antihypertensive effects by antagonizing their vasodilatory effects on the small arteriolar blood vessels.

Regarding the level of clinically significant interactions, majority $1160(62.7 \%)$ of the interactions were of moderate severity (type C). This is similar to previous reports. ${ }^{15,16,19,20}$ In this study, the prevalence of type X interaction (contraindication/ avoid drug combination) was $0.1 \%$, which was only found in one subject. This is comparable to $0.4 \%$ reported by Marquito et al. ${ }^{19}$

This finding is however at variance to that of Saleem et al ${ }^{18}$ who reported that $13.4 \%$ of potential DDIs observed in their study as avoid drug combination. The only type X interaction in this study occurred between intravenous calcium gluconate and intravenous ceftriaxone, which carries the potential risk of fatal particulate precipitation and deposition in the lungs and kidneys. The administration interval between intravenous ceftriaxone and any intravenous calcium-containing solutions (such as Ringer's solution, Hartman's solution, and intravenous calcium gluconate) must be separated by at least 48 hours apart.

A slightly higher proportion 960 (52\%) of potential DDIs was found to be from pharmacokinetic interactions. This is different from the reports in other studies where pharmacodynamic interactions were found to be predominant. ${ }^{16,20}$ Also, majority of the potential DDIs were of delayed onset, which is similar to report by Rama et al. ${ }^{16}$ The clinical significance of this is that the patients may not manifest the effects of DDI early, hence the need for long-term follow-up of such patients.

In addition, there was a statistically significant association between the number of prescribed medications and the eGFR (pre-ESRD and ESRD staging) for the respondents in this study with a $P$-value of 0.00000119 . This implies that the number of prescribed medications increases as the eGFR declines in advance CKD stage patients. This finding also agreed with previous studies by Sgnaolin et al, ${ }^{15}$ Rama et al, ${ }^{16}$ and Marquito et al. ${ }^{19}$ Furthermore, there was also a statistically significant association between the number of prescribed medications and the occurrence of DDIs among the respondents with a $P$-value of 0.00002421 . This implies that as the number of prescribed medications increases, the chances for occurrence of DDIs increases among these CKD patients. About $77.8 \%$ of those CKD patients taking around 1-5 medications are at risk of developing DDIs (compared to the value of $50 \%$ in previous reference literature) while $99.05 \%$ of those taking around 6 or more $(\geq 6)$ medications are at risk of developing drug-drug interactions (compared to the value of $100 \%$ in previous reference literature). ${ }^{15,16,19,20}$

The limitation of this study was that potential DDIs detected in this study were theoretically discovered and may not manifest clinically. Also, the Medscape drug interaction checker used in this study did not take into consideration the prescribed dose, frequency of administration, route of administration, and duration of medication use.

However, this study has brought to limelight the magnitude of potential DDIs among CKD patients and the need to take proactive steps to reduce these additional burdens on our patients.

\section{Conclusion}

The number of prescribed medications increases as the eGFR declines in advance CKD stage patients. The practice of polypharmacy and prevalence of potential DDIs are high among these CKD patients. Most of these interactions have moderate severity and delayed onset, hence the need to follow-up these patients after prescription in order to reduce associated morbidity, mortality, and health care costs. Physicians and clinical pharmacists should utilize available interaction software in order to detect and avoid harmful DDIs in our patients.

\section{Recommendations}

1. Physicians and clinical pharmacists should make use of available interaction software to check all prescribed medications for the presence of potentially significant/ harmful interactions.

2. Cordial integrated relationship between health care professionals (nephrologists/physicians, nephrology nurses, and clinical pharmacists) should be encouraged in order to optimize CKD patients' care and to reduce the occurrence of harmful drug interactions in them.

3. Whenever clinical pharmacists who are involved in the rational dispensing of CKD patients' medications detect the presence of any harmful potential DDIs in their prescription, he/she should alert the doctor(s) involved and inform them of the impending problem so that feasible alternative prescriptions can be made and the error corrected as this will involve more cost in pharmacist and doctor's time, but the hospital should make this mandatory. This approach will save lives and reduce morbidity, mortality, frequency of hospitalization, length of hospital stay, and health care costs. 


\section{Acknowledgment}

The authors specially thank Prof SO Olayemi (Department of Pharmacology \& Therapeutics) and Dr RW Braimoh (Department of Internal Medicine), University of Lagos, Lagos State Nigeria, for their immense support, advice, and contribution toward the success of this research work.

\section{Disclosure}

The authors report no conflicts of interest in this work.

\section{References}

1. Fatoba ST, Oke JL, Hirst JA, O'Callaghan CA, Lesserson DS, Hobbs FD. Global prevalence of chronic kidney disease: a systematic review and meta-analysis. PLoS One. 2016;11(7):e0158765.

2. Kadiri S, Arije A. Temporal variations and meteorological factors in hospital admissions of chronic renal failure in South West Nigeria. West Afr J Med. 1999;18(1):49-51.

3. Akinsola W, Odesanmi WO, Ogunniyi JO, Ladipo GO. Diseases causing chronic renal failure in Nigerians - a prospective study of 100 cases. Afr J Med Med Sci. 1989;18(2):131-137.

4. Ulasi II, Ijoma CK, Onodugo OD, Arodiwe EB, Ifebunandu NA, Okoye JU. Towards prevention of chronic kidney disease in Nigeria: a community-based study in Southeast Nigeria. Kidney Int Suppl. 2013;3(2): 195-201.

5. Oluyombo R, Ayodele OE, Akinwusi PO, et al. A community study of the prevalence, risk factors and pattern of chronic kidney disease in Osun State, South West Nigeria. West Afr J Med. 2013;32(2):85-92.

6. Liu M, Li XC, Lu L, et al. Cardiovascular disease and its relationship with chronic kidney disease. Eur Rev Med Pharmacol Sci. 2014;18(19): 2918-2926.

7. Levin A, Hemmelgarn B, Culleton B, et al. Guidelines for the management of chronic kidney disease. CMAJ. 2008;179(11):1154-1162.

8. Keith DS, Nichols GA, Gullion CM, Brown JB, Smith DH. Longitudinal follow-up and outcomes among a population with chronic kidney disease in a large managed care organization. Arch Intern Med. 2004;164(6):659-663.

9. Anavekar NS, Pfeffer MA. Cardiovascular risk in chronic kidney disease. Kidney Int Suppl. 2004;92:111-115.

10. Babua C, Kalyesubula R, Okello E, et al. Cardiovascular risk factors among patients with chronic kidney disease attending a tertiary hospital in Uganda. Cardiovasc J Afr. 2015;26(4):177-180.

11. Edwards IR, Aronson JK. Adverse drug reactions: definitions, diagnosis, and management. Lancet. 2000;356(9237):1255-1259.

12. Pirmohamod M, James S, Meakin S, Green C, Scott AK, Walley TJ. Drug-drug interaction as cause of admission to hospital: prospective analysis of 18820 patients. Br Med J. 2004;329:5-19.
13. Lazarou J, Pomeranz BH, Corey PN. Incidence of adverse drug reactions in hospitalized patients: a meta-analysis of prospective studies. JAMA. 1998;279(15):1200-1205.

14. Al-Hajje AH, Atoui F, Awada S, Rachidi S, Zein S, Salameh P. Drugrelated problems identified by clinical pharmacist's students and pharmacist's interventions. Ann Pharm Fr. 2012;70(3):169-176.

15. Sgnaolin V, Sgnaolin V, Engroff P, De Carli A, Figueiredo AE. Assessment of used medications and drug-drug interactions among chronic renal failure patients. Sci Med. 2014;24(4):329-335.

16. Rama M, Viswanathan G, Acharya LD, Attur RP, Reddy PN, Raghavan SV. Assessment of drug-drug interactions among renal failure patients of nephrology ward in a South Indian tertiary care hospital. Indian $J$ Pharm Sci. 2012;74(1):63-68.

17. Al-Ramahi R, Raddad AR, Rashed AO, et al. Evaluation of potential drug-drug interactions among Palestinian hemodialysis patients. $B M C$ Nephrol. 2016;17:96.

18. Saleem A, Mashood I, Khan TM. Clinical relevancy and determinants of potential drug-drug interactions in chronic kidney disease patients: result from a retrospective analysis. Integr Pharm Res Pract. 2017;6:71-77.

19. Marquito AB, Fernandes NM, Colugnati FAB, de Paula RB. Identifying potential drug interactions in chronic kidney disease patients. $J$ Bras Nefrol. 2014;36(1):26-34.

20. Hedge S, Udaykumar P, Manjuprasad MS. Potential drug interactions in chronic kidney disease patients. A cross-sectional study. Int J Recent Trends Sci Technol. 2015;16:56-60.

21. Shad MU, Marsh C, Preskorn SH. The economic consequences of drug-drug interaction. J Clin Psychopharmacol. 2001;21(1):119-120.

22. Moura CS, Acurcio FA, Belo NO. Drug-drug interactions associated with length of hospital stay and cost of hospitalization. J Pharm Pharm Sci. 2009;12(3):266-272.

23. Fernández-Llimós F, Tuneu L, Baena MI, Garcia-Delgado A, Faus MJ. Morbidity and mortality associated with pharmacotherapy. Evolution and current concept of drug-related problems. Curr Pharm Des. 2004;10(31):3947-3967.

24. Mason NA. Polypharmacy and medication-related complications in the chronic kidney disease patient. Curr Opin Nephrol Hypertens. 2011;20(5):492-497.

25. Cardone KE, Bacchus S, Assimon MM, Pai AB, Manley HJ. Medicationrelated problems in chronic kidney disease. Adv Chronic Kidney Dis. 2010;17(5):404-412.

26. Stemer G, Lemmens-Gruber R. Clinical pharmacy activities in chronic kidney disease and end-stage renal disease patients: a systematic literature review. BMC Nephrol. 2011;12:35.

27. International Society of Nephrology. Kidney disease improving global outcome (KDIGO) 2012 clinical practice guideline for evaluation and management of CKD. Kidney Int Suppl. 2013;3:1-150.

28. Roblek T, Trobec K, Mrhar A, Lainscak M. Potential drug-drug interactions in hospitalized patients with chronic heart failure and chronic obstructive pulmonary disease. Arch Med Sci. 2014;10(5):920-932.

29. Elam-Org S, Sitprija V. Co-morbidities in patients with end stage renal disease in developing countries. Artif Organs. 2002;26(9):753-756.
Clinical Pharmacology: Advances and Applications

\section{Publish your work in this journal}

Clinical Pharmacology: Advances and Applications is an international, peer-reviewed, open access journal publishing original research, reports, reviews and commentaries on all areas of drug experience in humans. The manuscript management system is completely online and includes a very quick and fair peer-review system, which is all easy to use.

\section{Dovepress}

Visit http://www.dovepress.com/testimonials.php to read real quotes from published authors. 